\title{
Die Grenzen der Gesellschaften
}

\section{Markus Schroer}

\begin{abstract}
Der Beitrag widmet sich verschiedenen Gesellschaftsformen und ihren jeweiligen Grenzen. Es wird gezeigt, dass das Werden von Gesellschaften ohne die Berücksichtigung der Grenzen, die sie jeweils für sich bestimmen, nicht darstellbar ist. Statt jedoch von einem zunehmenden Auf- oder Abbau von Grenzen auszugehen, wird dafür plädiert, den Formwandel der Grenzen stärker als bisher in den Blick zu nehmen. Als elementare Aufgabe einer Soziologie der Grenze wird das Ziel ausgegeben, den aus verschiedenen Modi von Grenzziehungen resultierenden Gesellschaftstypen je verschiedene Grenztypen zuzuordnen.
\end{abstract}

\section{Schlagwörter}

Grenze, Gesellschaft, Entgrenzung, Abschottung, Formwandel

\section{Einleitung}

Keine Gesellschaft kommt ohne Grenzziehungen aus. Die erste von ihr vollzogene Grenzziehung ist diejenige, mit der sie sich von etwas anderem unterscheidet. Gesellschaft ist damit selbst das Produkt einer Grenzziehung. Damit die Gesellschaft sie selbst sein kann, muss sie sich von etwas anderem abgrenzen (lassen). In der sozialwissenschaftlichen Literatur wird die Gesellschaft klassischerweise der Natur (vgl. Rehberg 2008), der Kultur (vgl. Rehberg 1985), dem Staat (vgl. Luhmann 1994), dem Individuum (vgl. Schroer 2001a), der Gemeinschaft (vgl. Tönnies 1979) und anderen Gesellschaften (vgl. Immerfall 1995) gegenübergestellt, um anschließend über die Beziehungen nachzudenken, die zwischen den so unterschiedenen Größen bestehen. Erst durch diese Trennung werden beide Seiten der Unterscheidung in eine gewisse Spannung zueinander gebracht. Dabei handelt es sich nicht um rein epistemologische Grenzziehungen, die eine arbeitsteilig verfahrende wissenschaftliche Erforschung ermöglichen soll, sondern um tief in die moderne Gesellschaft eingesenkte Denkkategorien, die das Handeln der Menschen und die soziale Praxis prägen. Sie zeigen, dass in einem unübersichtlichen Feld von Möglichkeiten einzelne Bereiche eingegrenzt und von anderen abgegrenzt werden, um der Komplexität des Gegebenen eine Ordnung abzuringen, die auf der Einführung solcher Dualismen beruht. Grenzziehungen bringen also Ordnungen hervor und jede Ordnung verdankt sich bestimmter Grenzziehungen. Je nachdem, wie diese gezogen werden, entsteht eine je spezifische (gesellschaftliche) Ordnung. Im vorliegenden Beitrag geht es darum, das Verhältnis von Gesellschaften und ihren Grenzen zu analysieren. Dabei stellen sich grundsätzliche Fragen: Wo verlaufen die Grenzen der Gesellschaften? Welche Art Grenzen ziehen Gesellschaften? Wer legt die Grenzen der Gesellschaften fest? Gibt es eine klar zu bestimmende Grenze, an der Gesellschaft aufhört und etwas anderes beginnt? Was wäre dieses andere?

Um diesen Fragenkomplex bearbeiten zu können, geht es im Folgenden um die nähere Bestimmung der beiden Begriffe Grenze und Gesellschaft (1). Dabei werden beide nicht isoliert behandelt, sondern von Anfang an aufeinander bezogen. Es geht also nicht einerseits um Gesellschaft und andererseits um Grenzen, sondern um die „Grenzen der Gesellschaft“ (Mauss 1975, S. 134; Lévi-Strauss 1967, S. 321). Der darauffolgende historische Abriss (2) zeigt, dass Jäger- und Sammlergesellschaften, Agrargesellschaften und Nationalgesellschaften nicht nur 
über Grenzen verfügen, sondern das Produkt von Grenzziehungen sind, die sie selbst vornehmen. Im Anschluss daran werden verschiedene Gesellschaftsentwürfe (globale Gesellschaft, Weltgesellschaft, Netzwerkgesellschaft, „Liquid Modernity“) vorgestellt (3) und kritisiert (4). Schließlich wird die von den neuen Gesellschaftsformen geteilte Vorstellung einer wachsenden Indifferenz gegenüber physischen Räumen und territorialen Grenzen mit der aktuellen These einer weltweiten Wiederkehr der Grenzen konfrontiert (5). Der Beitrag plädiert abschließend (6) im Sinne einer elaborierten Grenzforschung dafür, den Suggestionen einer grenzenlosen Gesellschaft auf der einen und von sich einmauernden Gesellschaften auf der anderen Seite nicht zu erliegen, sondern den Wandel der Grenzformen in den Mittelpunkt der grenzsoziologischen Forschung zu stellen. Elementare Aufgabe einer Soziologie der Grenze wäre es, den aus verschiedenen Modi von Grenzziehungen resultierenden „Gesellschaftstypen“ (vgl. Tenbruck 1989a; Popitz 1995, S. 130) je verschiedene Grenztypen zuzuordnen.

\section{Gesellschaften und ihre Grenzen}

Der sozialen Morphologie von Emile Durkheim, Marcel Mauss und Maurice Halbwachs (vgl. Halbwachs 2002; Durkheim 2010; Mauss 2010; Schroer 2018a) verdanken wir die zentrale Einsicht, dass der Terminus Gesellschaft nicht für eine rein abstrakte Vorstellung steht, die ausschließlich in den Köpfen der Menschen vorkommt, sondern ein durchaus konkretes Gebilde bezeichnet: „Keine Gesellschaft, die sich nicht auch in ihren räumlichen Umrissen zeigte, nicht eine Ausdehnung und materielle Unterlage hätte.“ (Halbwachs 2002, S. 23) Gerade ihre morphologische Struktur bestimmt die jeweilige Form einer Gesellschaft:

„Je nachdem, ob die Bevölkerung mehr oder weniger umfangreich und mehr oder weniger dicht ist, je nachdem, ob sie in den Städten konzentriert oder über das Land zerstreut, je nach Bauweise der Städte und der Häuser, je nachdem, ob der von der Gesellschaft eingenommene Raum mehr oder weniger ausgedehnt ist, je nachdem, welches ihre Grenzen sind und welches die Verkehrswege, die sie durchziehen, etc., ist das soziale Substrat ein verschiedenes. “ (Durkheim 2010, S. 182)

Gesellschaften schreiben sich förmlich in den Raum ein, nehmen unterschiedliche Gestalt an, bleiben aber niemals eine bloße Idee. Dabei zeichnen sie sich vor allem auch durch ihre Grenzziehungen aus, mit denen sie sich überhaupt erst konstituieren:

„Gesellschaften [...] sind soziale Einheiten. Wo immer Menschen sich zusammentun, wo sie ihr Verhalten alltäglich einander orientieren, entstehen soziale Einheiten: WildbeuterHorden, Familien, Sippen, Städte, bis hin zum Gesangsverein. Vergesellschaftung gehorcht einem zellenbildenden Prinzip. Soziale Einheiten sind leicht zu erkennen, weil sie Wert darauf legen, sich erkennbar zu machen. Sie ziehen Grenzen zwischen Innen und Außen, Drinsein und Draußensein. Wer als Zugehöriger anerkannt wird, muß über bestimmte Qualitäten verfügen, angeborene wie Geschlecht oder Herkunft, oder erworbene wie bestimmte Leistungen oder Bewährungen. Vergesellschaftung bedeutet, daß Menschen in Strukturen von Zugehörigkeiten leben. Also in Ein- und Ausgrenzungen. Das zellenbildende Prinzip der Vergesellschaftung ist ein Prinzip der Grenzziehung.“ (Popitz 1995, S. 126f.)

Diese Auffassung von Gesellschaften ist für unseren Zusammenhang zentral, da hier Grenzziehungen als fundamentale Basisoperation von Gesellschaften angesehen werden. Gesellschaften ebenso wie andere soziale Einheiten bestimmen durch Ein- und Ausschließungen über Zugehö- 
rigkeit und Nichtzugehörigkeit. In diesem Sinne argumentiert auch Gesa Lindemann, wenn sie davon ausgeht, dass jede Vergesellschaftung auf einer Grenzregulierung beruht, „durch die entschieden wird, wer in den Kreis personaler Akteure gehört, die den Prozeß der Vergesellschaftung tragen, und was aus diesem Kreis ausgeschlossen ist." (Lindemann 2004, S. 26f.; vgl. auch Lindemann in diesem Band) Die Bestimmung des Gesellschaftsbegriffs von Heinrich Popitz enthält darüber hinaus den gleichermaßen zentralen Hinweis, dass soziale Einheiten selbst daran gelegen ist, sich erkennbar zu machen. Damit ist ein wichtiger Einspruch gegen ein Verständnis von Gesellschaft formuliert, das diese für ein nur schwer fassbares, weil nicht sichtbares, nur abstrakt beschreibbares Phantom hält, das „man nur vom Hörensagen, aus dem Fernsehen oder der Zeitung “ (Uzarewicz 2016, S. 60) kennt. Sich erkennbar machen zu wollen, heißt nichts anderes als Sichtbarkeit anzustreben (vgl. Schroer 2014). Eine sichtbare Gestalt erhalten Gesellschaften wie andere soziale Einheiten etwa durch das bei Durkheim angesprochene Bauen von Unterkünften, so dass sich von der Geburt der Gesellschaft aus der Praxis des Bauens sprechen ließe. Von den Anfängen der Menschheitsgeschichte bis heute machen sich verschiedene Gesellschaftstypen an ihrer jeweiligen Architektur sicht- und erkennbar (vgl. Delitz 2010). Von der „Urhütte bis zum Wolkenkratzer“ (Klotz 1991) stehen die verschiedenen Bauten und Baustile für jeweils bestimmte Gesellschaftsformen. Schon die Zeltarchitektur vormoderner Gesellschaften zeigt, „dass bereits die frühen Menschen alle sich bietenden Möglichkeiten der Gestaltung und Aneignung von Raum kreativ genutzt haben.“ (Trebsche et al. 2010, S. 19) Zu dieser aktiven Gestaltung von Räumen gehört immer auch die Notwendigkeit von Grenzziehungen, die Räume erst hervorbringen (vgl. Schroer 2019a).

Die von Popitz darüber hinaus thematisierten „Zugehörigkeitsgrenze[n]“ (Simmel 1992, S. 701) spielen auch in Stein Rokkans grundlegender Arbeit über den Aufbau von Staat und Nation (vgl. Rokkan 2000) eine tragende Rolle. Rokkan unterscheidet zwischen

„dem physischen Raum zum einen und dem sozialen und kulturellen zum anderen. Wir können den einen als den geographischen Raum, den anderen als den Mitgliedschaftsraum bezeichnen. Die Mitgliedschaftsgrenze ist gewöhnlich viel unüberwindbarer als die geographische Grenze.“ (ebd, S. 135, Herv. i. O.; vgl. Bös 2000)

Mit dem letzten Satz spricht Rokkan an, dass das Überschreiten der Grenze zu einem staatlichen Territorium für einen Touristen, Händler oder Gelegenheitsarbeiter sich zwar relativ leicht bewerkstelligen lässt, der Grenzübertritt aber nicht mit dem Erwerb von Mitgliedschaft einhergeht. Während Rokkan dabei noch an die Mitgliedschaft in einer „Kerngruppe“ denkt (ebd., S. 135), kann man im Anschluss an Popitz von einer Vielzahl sozialer Einheiten ausgehen, die nicht über einheitliche, sondern diverse Zugehörigkeitsregeln verfügen, die der Einzelne erfüllen muss, um dazugehören zu können. Das bedeutet, dass sich Gesellschaften nicht nur durch eine geographische Grenze nach außen, sondern durch vielerlei soziale Grenzziehungen im Innern bzw. durch Binnengrenzen auszeichnen. Sie sind durchsetzt von Grenzziehungen zwischen oben und unten, arm und reich usw., die sich zwar nicht zwangsläufig materialisieren müssen, um wirksam zu sein, dies aber durchaus oft tun - nicht zuletzt aufgrund des von Popitz betonten Strebens nach Sichtbarkeit. Wohl aber auch deshalb, weil die „sozialen Begrenzungsprobleme durch ihre Verräumlichung“ eine „unvergleichliche Festigkeit und Anschaulichkeit“ erlangen, worauf bereits Georg Simmel ausdrücklich hinweist (Simmel 1992, S. 699; vgl. Schroer 2006, S. 68f.):

„So ist eine Gesellschaft dadurch, daß ihr Existenzraum von scharf bewußten Grenzen eingefaßt ist, als eine auch innerlich zusammengehörige charakterisiert, und umgekehrt: 
die wechselwirkende Einheit, die funktionelle Beziehung jedes Elements zu jedem gewinnt ihren Ausdruck in der einrahmenden Grenze. “ (Simmel 1992, S. 694)

Da Gesellschaften demnach nicht als homogene Einheit vorstellbar sind, sie sich vielmehr in zahlreiche soziale Einheiten, Milieus und Gruppierungen unterteilen bzw. ausdifferenzieren, erweisen sie sich geradezu als - mitunter heiß laufende - Generatoren von Grenzen, die sich auch räumlich niederschlagen, wenn sie Regionen, Zonen, Gebiete und Areale voneinander abgrenzen. Gesellschaften haben also keine Grenzen von vornherein, sondern sind Produkt einer Grenzziehung, die sie von etwas anderem unterscheidet - etwa von der Natur bzw. der Umwelt. Haben sie sich durch eine Grenzziehung nach außen erst einmal konstituiert, werden Binnengrenzen gezogen, die auch auf die Zugehörigkeits- wie auf Abgrenzungsbedürfnisse der Menschen reagieren, die für Simmel einen Grundmodus menschlichen Daseins ausmachen (ebd., S. 855; vgl. Schroer 2001a, S. 311ff.). Jede Gesellschaftsform will über die Art und Weise ihrer Grenzziehungen festlegen und sichtbar machen, wer und was zu ihr gehört und wer oder was nicht, um sich damit - ganz im Sinne von Popitz - als identifizierbare Einheit erkennbar zu machen. Da sich Gesellschaften über ihre jeweiligen Grenzziehungen definieren und zu stabilisieren versuchen, wachen sie streng über der Einhaltung dieser Grenzen. Allerdings geht es nur in Ausnahmefällen um die Verteidigung von ein für alle Mal gezogenen Grenzen. Bezeichnend ist vielmehr, dass immer wieder neu vorzunehmende Grenzziehungen an der Tagesordnung sind. Grenzen werden errichtet, verteidigt, verlagert, verschoben, abgebaut und neu gezogen und sind damit eher dynamische denn starre Gebilde (vgl. Bös/Zimmer 2006). Dabei ist keineswegs nur an die politischen Grenzen von Nationalstaaten zu denken. Ob Körperraum, Wohnraum oder nationalstaatlicher Raum (vgl. Schroer 2006): Immer geht es darum, den Zugang zu diesen Räumen zu limitieren, ihn nur unter bestimmten Bedingungen und Auflagen zu erlauben. Jeder dieser Räume umgibt sich mit Grenzen, die den wohl dosierten Austausch mit der Umwelt erlauben und den ungehinderten Zutritt unterbinden können sollen: die Haut, die Tür und der Schlagbaum. So unterschiedlich diese Grenzen im Einzelnen auch sein mögen, gemeinsam ist ihnen die Funktion der Selektion (vgl. Lotman 2010): Sie alle sorgen für geregelte Übergänge in Räume, die nicht jedermann offenstehen. Während den einen Einlass gewährt wird, werden die anderen abgewiesen. Dabei spielt die Materialität der Grenze keine geringe Rolle für den jeweiligen Charakter der Grenze: Weiche Formen der Begrenzung wie etwa Membrane unterscheiden sich deutlich von harten Abschlüssen wie etwa Betonmauern (vgl. Schroer 2018b; 2019b). Insofern ließe sich jede Form von Gesellschaft mit den jeweiligen Grenzziehungen in Beziehung setzen, die sie hervorrufen und deren Produkt sie gleichzeitig sind.

\section{Gesellschaftstypen und ihre Grenzen}

Die Geschichte der Konstituierung von Gesellschaften durch das Ziehen von Grenzen kann bis in die Ursprünge der Menschheitsentwicklung zurückverfolgt werden: „Am Anfang steht der Zaun. Tief und begriffsbestimmend durchwirken Zaun, Hegung, Grenze die von Menschen geformte Welt." (Trier 1943, S. 232; vgl. Freyer 1965, S. 23) Grenzen sind also keineswegs eine Erfindung der Nationalstaaten. Zwar haben wir es bei den historischen Vorläufern noch nicht mit jenen klar gezogenen Grenzlinien zu tun, die spätestens seit dem 19. Jahrhundert zwischen den Nationalstaaten gezogen werden. Doch die Gleichsetzung dieser spezifischen Form der Grenze zur Grenze schlechthin birgt die Gefahr, den Blick auf deren zahlreiche Vorläufer zu verstellen (vgl. auch Schmieder in diesem Band). Nach allem, was wir heute 
wissen, hat der Mensch in der Frühzeit zwar nicht in Höhlen gehaust, sondern „in künstlich errichteten Schutzbauten“ (Leroi-Gourhan 1980, S. 396) wie etwa Zelten und Hütten, die am Eingang der Höhle errichtet wurden. Doch schon in dieser frühen Periode der Menschheitsgeschichte wurde ein „Wohnraum gegen das Chaos der Umgebung abgegrenzt“ (ebd., S. 397), der Lebensraum in einen „Verdauungs- und Fortpflanzungsraum (die Hütte)“ (Flusser 2006, S. 279) einerseits und einen „Jagdraum“ (ebd.) andererseits unterteilt und damit eine „Grenze zwischen bewohntem und unbewohntem Raum“ (Eßbach 1999, S. 88) gezogen. Bereits hier haben wir es also mit der Grenzziehung zwischen einem Innen- und einem Außenraum zu tun. Während dem Außenraum die Grundlagen für die Ernährung abgerungen werden, dienen die Innenräume primär als Schutzräume. Dieser Schutz wird benötigt, „weil der Mensch zunächst einmal den Bedürfnissen seines Organismus genüge tun “ muss (Malinowski 2005, S. 76):

„Zur Ernährung und Behausung, zur Kleidung und zum Schutz vor Kälte, Wind und Wetter muß er Einrichtungen treffen und Tätigkeit entfalten. Er muß sich selber schützen und diesen Schutz gegen äußere physische, menschliche und tierische Feinde und Gefahren organisieren.“ (ebd.)

Über die Funktion des Schutzes hinaus spielen die ersten Behausungen aber auch eine elementare Rolle für die Stabilisierung von Mitgliedschaft in einem sozialen Verband. Dieter Claessens betont den „imperativischen Charakter“ (Claessens 1980, S. 71), der vom vertrauten Raum einer einfachen Lagerstätte wie etwa einer Höhle ausgeht:

„[D]as in diesen Raum eintretende Gruppenmitglied erfährt durch den Raum, daß es Mitglied ist; es wird in diesem bestätigt und weiter geprägt, und gleichzeitig resultieren aus dieser Aufforderung auch alle jene Anweisungen, die sich aus dem Gruppenleben entwickelt haben und sozusagen im Raum sich sicht- und fühlbar eingenistet haben“ (ebd.).

In diesem Zeitraum fallen physischer Raum und Mitgliedschaftsraum also noch zusammen: Wer den Raum betreten darf, gehört auch dazu. Zusätzlich „zieht das Verwandtschaftssystem klare Grenzen zwischen Personen, die zur Gemeinschaft gehören, und solchen, die ausgeschlossen sind." (Rokkan 2000, S. 135) Obwohl unter Anthropologen über die Frage des Territorialitätsbezugs einfacher Gesellschaftsformen durchaus gestritten wird, herrscht weitgehend Einigkeit darüber, dass „viele primitive Gesellschaften sich aus Familien, Horden und Lineages zusammensetzen, die sehr wohl fest in abgegrenzten Territorien zusammenleben“ (Service 1977, S. 99, Herv. i. O.). Wenngleich dies auch schon für nicht-sesshafte, nomadische Völker gilt - „[a]uch wandernde Verbände der Jäger-Sammler-Stufe haben ein privilegiertes Territorium“ (Gehlen 1964, S. 15) -, verstärkt sich offenbar der exklusive Anspruch auf ein Territorium im Laufe der sozialen Evolution:

„Mit der neolithischen Revolution, mit der Ausbreitung der Landwirtschaft und der Gründung von Städten, wurde die Beständigkeit des Besitzes und das Markieren von territorialen Grenzen zu einem grundlegenden Anliegen gesellschaftlicher Organisation." (Rokkan 2000, S. 135; vgl. Løvschal 2014)

Dies betont auch Durkheim in seiner Beschreibung der einfachen Gesellschaften, die sich aus weitgehend homogenen Segmenten wie Horden, Klans, Stämmen oder Familien zusammensetzen. Jeder Klan lebt streng gesondert vom nächsten. Die von ihnen bestellten Felder lagen niemals direkt nebeneinander, sondern waren durch frei liegende Flächen voneinander getrennt, die nicht für die Landwirtschaft genutzt werden durften: 
„Jedes Feld mußte [...] von einer Umfriedung umgeben sein, die es von dem Besitztum der anderen Familien streng abschloß. Diese Umfriedung war keine steinerne Mauer: sie war ein Streifen Erde, einige Fuß breit, der unbebaut bleiben mußte, und den der Pflug nie berühren sollte. Dieser Zwischenraum war geheiligt. [...] An gewissen und bestimmten Tagen des Monats und des Jahres machte der Familienvater einen Rundgang um seine Felder, indem er diese Linie verfolgte; er trieb die Opfertiere vor sich hin, sang Hymnen und brachte Opfer dar. [...] Der Weg, den die Opfertiere und der betende Mann verfolgt hatten, war die unverletzliche Grenze der Behausung. In diese Linie legte der Mensch in gewissen Zwischenräumen große Steine oder Pfähle, die man Grenzsteine nannte.“ (Coulanges 1996, S. 67f.; vgl. Durkheim 1991, S. 211f.; Schroer 2006, S. 51ff.; Schroer i. E.)

Als oberstes Gebot galt in dieser Zeit: „Überschreite nicht den Grenzstein“ (Coulanges 1996, S. 69).

Spätestens an diesem Punkt der historischen Entwicklung stoßen wir auf den engen Zusammenhang von Grenze und Eigentum bzw. Eigentumsrecht. Die mit der Sesshaftigkeit einhergehende Ausbreitung von Ackerbau und Viehzucht führt zur sukzessiven Aufteilung der Erdoberfläche in Kleinterritorien, deren Grenzsteine das Eigentum an Grund und Boden sowohl physisch als auch symbolisch markieren. In der politischen Theorie und Rechtsgeschichte wird von John Locke über Jean-Jacques Rousseau bis hin zu Carl Schmitt der „Akt des Einzäunens“ (Bühler 2014, S. 70) als fundamentaler Einschnitt gewertet, der den Übergang vom Natur- zum Gesellschaftszustand definiert. Die exklusive Inbesitznahme von einem Stück Land und dessen Verteidigung setzt zwar deutlich vor der Konstituierung moderner Staaten ein: „Die Menschen entwickelten schon früh ein Repertoire von Signalen, um Besitz oder Besetzung eines Territoriums zu identifizieren, und organisierten sich, um Eindringlinge zurückzudrängen." (Rokkan 2000, S. 141) Dennoch besteht weithin Einigkeit darüber, dass die Exklusivität des Territoriums und die „ausgeprägte Sensibilität für das Gebiet, in dem die Gesetze gelten sollen und das zu verteidigen ist“ (Service 1977, S. 99), im Laufe der gesellschaftlichen Evolution zunimmt und erst mit der Staatenbildung zur vollen Entfaltung gelangt. Der Hinweis auf die Gesetze ist wichtig für das Verständnis der Herausbildung von Nationalstaaten. Schon für Montesquieu ist der Staat eine „Gesellschaft, in der es Gesetze gibt.“ (Montesquieu 1951, S. 212) Auch für Carl Schmitt gehören die juristische Ordnung und das Territorium unauflöslich zusammen. Für ihn ist das Recht die „Einheit von Ordnung und Ortung“ (Schmitt 1997, S. 13).

Der Rückblick auf frühere Gesellschaftsformen zeigt gleichwohl, dass nicht erst moderne Staaten, sondern schon ihre Vorgänger über Territorien und Grenzen verfügten. An Rokkan anschließend, lässt sich auch die Gründung von Städten als zentraler Ausdruck für den wachsenden Bedarf verstehen, angehäufte Besitztümer durch das Markieren von territorialen Grenzen zu schützen:

„Die entscheidende Innovation der Stadt war, daß sie starke Grenzen für eine differenzierte Kontrolle von Transaktionen ausbildete. Ganz gleich, ob sie physisch von einer Mauer umgeben war oder nicht: Die Stadt des Altertums war eine rituelle und rechtliche Gemeinschaft, die strenge Kontrolle über ihre Mitglieder ausübte, während sie gleichzeitig ihre Grenzen für Export und Import sowohl von Personen [...] als auch von Waren offenhielt.“ (Rokkan 2000, S. 136) 
Selbst dort also, wo es zur Errichtung von Mauern kommt, die geradezu als Sinnbild für radikale Abschottung stehen, geht es nicht um die gänzliche Verhinderung des Austausches von Waren und Personen, sondern um dessen Organisation. Das war selbst beim Limes schon so (vgl. Scheuerbrandt 2009).

Neu an der Entstehung der modernen Staaten ist deshalb nicht, dass es überhaupt zur Besetzung und Verteidigung von Territorien und der Errichtung von Grenzen kommt. Neu ist vielmehr die Etablierung eines spezifischen Grenztyps, der bis heute unser Bild von Grenzen prägt: „Die Idee einer linearen Grenze entstand ab dem 14. und 15. Jahrhundert, mit der Entwicklung des modernen Staates. [...] Der moderne Staat beansprucht eine lineare Grenze - zumindest als Begriff - allein schon aufgrund seines territorialen Wesens." (Raffestin 2010, S. 60) Die sich im 19. Jahrhundert endgültig etablierenden Nationalgesellschaften zeichnen sich durch klare Grenzziehungen gegenüber anderen Nationalgesellschaften aus. Auf dem Höhepunkt der Verbreitung der nationalstaatlichen Ordnung stoßen Staaten unmittelbar aufeinander - nur durch eine Grenzlinie voneinander geschieden. An diesen Grenzlinien wird die Kontrolle über das Territorium ausgeübt, der Zugang von Personen und Waren gewährt oder verweigert (vgl. Schroer 2019b). Die ehemals bestehenden Zwischenräume und Pufferzonen sind in dieser Zeit auf ein Nichts zusammengeschrumpft:

„Wie die Flur als ganze, sogar viel sichtbarer als sie, grenzt sich jeder bebaute Acker von selber, als Muster aus Furchen, als Saatfläche, als Ährenfeld, als Stoppel. Denn jeder Ort und jede Verortung an ihm setzt auch den Nachbarn und setzt die Grenze gegen ihn. Wenn die Nachbarn näher rücken und die Flur endgültig aufgeteilt ist, werden die Grenzen scharf.“ (Freyer 1965, S. 23)

Weil die Populationen der einzelnen sozialen Einheiten größer werden und dadurch immer mehr in die unmittelbare Nähe der anderen Einheiten gelangen, schrumpfen die ehemaligen Grenzgebiete auf eine „scharf“ gezogene Grenzlinie zusammen. Der so beschriebene Vorgang stützt die These Friedrich Tenbrucks,

„daß die gesellschaftliche Entwicklung im Grunde kein Binnenvorgang ist, sondern aus raumgreifenden Expansionen und zwischengesellschaftlichen Verbindungen hervorgeht, wie sie sich durch Eroberung, Unterwerfung, Kolonisierung, Vereinigung, Verflechtung oder andere Formen der Ausdehnung [...] ergeben.“ (Tenbruck 1989b, S. 432)

Entgegen dem vor allem in systemtheoretischen Differenzierungstheorien vorherrschenden Grundgedanken, dass sich die gesellschaftliche Entwicklung durch den Wandel der Differenzierungsformen (von der segmentären über die stratifikatorische zur funktionalen Differenzierung) „innerhalb der Gesellschaft und als Binnengeschehen“ (Tyrell 2005, S. 39, Herv. i. O.) vollzieht, gelangt Tenbruck zu einem ganz anderen Ergebnis:

„Stets und überall sind es zwischengesellschaftliche Verhältnisse und Veränderungen durch raumgreifende Vorgänge gewesen, die eine gesellschaftliche Entwicklung mittels Bildung jeweils größerer Einheiten ermöglicht haben. Selbst bei räumlicher Isolierung wird das Leben in einer Gesellschaft durch die Orientierung an ihren Außenlagen bestimmt, die als wichtiger Teil der Umwelt bewußt sind und die Binnenstruktur beeinflussen.“ (Tenbruck 1989b, S. 434f.)

Tenbruck widerspricht grundsätzlich der Annahme eines „Ein-Gesellschaft-Modells“ und stimmt darin mit Cornelius Castoriadis überein, der ebenfalls nicht von einer „Produktion diverser Exemplare der Wesenheit ,Gesellschaft', sondern von der Schöpfung eines anderen 
Typus des Gesellschaftseins (einer anderen Form/Gestalt, eines anderen Aspekts/Sinns: eidos)“ (Castoriadis 1984, S. 607, Herv. i. O.) ausgeht: „Die Gesellschaft als solche ist eine Form, und jede bestehende Gesellschaft ist eine besondere, ja einzigartige Form. “ (Castoriadis 2010, S. 27) Schon bei Durkheim heißt es ausdrücklich:

„,[D]ie Gesellschaft' existiert nicht. Es gibt ,Gesellschaften', die sich in Gattungen und Arten einteilen lassen, ähnlich wie bei den Gewächsen und Tieren. [...] Heute [...] ist es offenkundig unmöglich, als wahr zu behaupten, daß es eine überall mit sich selbst identische menschliche Entwicklung gibt, und daß die Gesellschaften alle nur Variationen ein und desselben Typs sind.“ (Durkheim 1981, S. 35)

Als Beispiel für die von Tenbruck erwähnte Bildung größerer Einheiten käme in jüngerer Zeit etwa die Europäische Union in Frage, durch die die Vielfalt von Gesellschaften jedoch keineswegs aufgehoben wurde. Ganz im Gegenteil, es kommt nahezu parallel zum Zusammenschluss der Länder unter dem Dach der EU zur Gründung neuer Nationalstaaten und Abspaltungsbestrebungen einzelner Regionen innerhalb einzelner Nationalstaaten. Im Zuge der Globalisierungsdebatte der 1990er Jahre werden an die Stelle der Nationalstaaten - die bei Marcel Mauss noch als „die jüngste und vollkommenste Form des Lebens in Gesellschaften“ (Mauss 2017, S. 336) angesehen werden - immer wieder alternative Gesellschaftskonzeptionen gesetzt, die sich nach der Überzeugung ihrer Verfechter von physischen Räumen und territorialen Grenzen weitgehend unabhängig machen und die Bindung der Gesellschaft an den Nationalstaat überwinden (vgl. Schroer 2018b).

\section{Gesellschaft ohne Grenzen?}

Der schillernde Begriff der Globalisierung wird in weiten Teilen der Sozial- und Kulturwissenschaften als Entwicklung verstanden, die zum Bedeutungsverlust des Raumes, der Grenzen und des Nationalstaates führt (vgl. Schroer 2006, S. 185ff.). Globalisierung steht hier für einen weltweiten Prozess der Enträumlichung, Entgrenzung bzw. Deterritorialisierung (vgl. Robertson 1992; Giddens 1995; Schroer 2006, S. 195ff.). In dieser Perspektive erscheinen Grenzen rein negativ als unerwünschtes Hindernis und lästige Hürden, die der Durchsetzung einer ungehinderten Mobilität vorübergehend zwar noch im Wege stehen, schon bald aber verschwunden sein werden. Angesichts des weltweiten Austausches von Waren, Dienstleistungen und Informationen, der Entwicklung globaler Kapitalmärkte und zunehmender Migration über nationale Grenzen hinweg, sei die Vorstellung nicht mehr länger plausibel, „in geschlossenen und gegeneinander abgrenzbaren Räumen von Nationalstaaten und ihnen entsprechenden Nationalgesellschaften zu leben und zu handeln“ (Beck 1997, S. 44). Die Nationalstaaten drohen in dieser Sichtweise zunehmend die Kontrolle über die auf ihrem Territorium stattfindenden Aktivitäten zu verlieren und damit an Souveränität einzubüßen. Ihre immer auch Sicherheit versprechenden Grenzen hätten sich zunehmend als wirkungslos erwiesen, die eigene Bevölkerung und das eigene Territorium umfassend vor Einflüssen und Gefahren von außen zu schützen. In der Tat haben sich die radioaktiven Wolken nach dem Reaktorunglück in Tschernobyl ebenso wenig von Nationalstaatsgrenzen aufhalten lassen wie Krankheiten und Seuchen (BSE, Schweinegrippe, Ebola), die Finanzkrise oder der Terrorismus. Der Fall der Berliner Mauer, der Untergang der Sowjetunion und das Ende des Ost-West-Gegensatzes haben zudem gezeigt, dass Grenzen nicht nur mehr oder weniger mühelos überschritten, sondern sogar buchstäblich niedergerissen und zum Einsturz gebracht werden können - selbst wenn 
sie bis dato für viele als schier unüberwindbar gegolten haben. Aufgrund dieses umfassenden Versagens und Abbaus der Grenzen wurde eine „Borderless World“ (Ohmae 1990) in Aussicht gestellt, ein baldiges Ende des Nationalstaates prophezeit (vgl. Albrow 1998) oder eine „postnationale Konstellation“ (Habermas 1998) anvisiert:

„Gegenüber der territorialen Verankerung des Nationalstaats beschwört der Ausdruck ,Globalisierung' das Bild von anschwellenden Flüssen, die die Grenzkontrollen unterspülen und das nationale Gebäude zum Einsturz bringen können. Die neue Relevanz von Fließgrößen signalisiert die Verschiebung der Kontrollen aus der Raum- in die Zeitdimension. Die Verlagerung der Gewichte vom ,Beherrscher des Territoriums‘ zum ,Meister der Geschwindigkeit‘ scheint den Nationalstaat zu entmachten.“ (ebd., S. 103)

Unter diesen Bedingungen verliert auch die Gesellschaft ihre nationalstaatliche Basis. Den zahlreichen Neubeschreibungen von Gesellschaften, die an die Stelle der Nationalgesellschaften treten, ist bei allen sonstigen Unterschieden gemeinsam, dass sie nicht länger im nationalstaatlichen Rahmen verbleiben, sondern weit über diesen hinausführen oder ihn gleich ganz hinter sich lassen. Ob nun von „Globalisierung“ (Giddens 1995), der „Weltgesellschaft“ (Luhmann 1971; Stichweh 2000), der „Netzwerkgesellschaft“ (Castells 2001) oder der „Liquid Modernity“ (Bauman 2000) die Rede ist: Gemeinsam ist all diesen Konzepten, dass sie dafür werben, Gesellschaften nicht mehr länger mit den Nationalstaaten gleichzusetzen, wie dies in der Soziologie bisher - so die nahezu einhellige Kritik an ihrem methodologischen Nationalismus (Beck 1997, S. 46) - der Fall gewesen sei. Darüber hinaus vertreten sie übereinstimmend die These, dass Gesellschaften sich von räumlichen Grenzen zunehmend emanzipieren.

Niklas Luhmanns Konzept der „Weltgesellschaft“ (Luhmann 1971) verdankt sich seinem gesellschaftstheoretisch ambitionierten Versuch, Gesellschaft grundsätzlich antiterritorial zu denken. Das moderne Gesellschaftssystem hat gegenüber seinen Vorgängern derart an Komplexität und Heterogenität zugenommen, dass es „seine Teilsysteme nicht mehr durch gemeinsame (etwa territoriale) Außengrenzen integrieren “ kann (Luhmann 1973, S. 89), weshalb Gesellschaft „von jetzt ab nur noch als Weltgesellschaft möglich“ ist (ebd.) und dabei keine anderen Gesellschaften mehr neben sich duldet. Gegenüber der traditionellen Vorstellung einer Vielzahl menschlicher Gesellschaften gibt es Gesellschaft für den Bielefelder Systemtheoretiker nur noch im Singular, als Weltgesellschaft. Neben den „technischen Errungenschaften“ (Luhmann 1971, S. 60) ist die „Ausdifferenzierung des Wirtschaftssystems“ (Luhmann 1973, S. 89) die treibende Kraft dieser Entwicklung. Der internationale Geld- und Zahlungsverkehr macht vor nationalen Grenzen nicht länger halt. Doch dabei bleibt es nicht. Die grenzüberschreitenden Aktivitäten des Wirtschaftssystems machen Schule und infizieren auch die anderen Teilsysteme, die sich aus dem engen Korsett der territorialen Grenzen des Nationalstaats ebenfalls befreien. Mit Ausnahme des Politik- und Rechtssystems operieren alle Funktionssysteme „unabhängig von Raumgrenzen“ (Luhmann 1997, S. 166). Mit Luhmann erhält man damit einen von allen ,alteuropäischen“ Annahmen weitgehend gereinigten Gesellschaftsbegriff, der sich um Raum, Grenze, Territorialität, Erde, Land, Boden und Materialität nicht länger schert. Unter Gesellschaft wird nur mehr „das jeweils umfassendste System kommunikativer Beziehungen zwischen menschlichen Erlebnissen und Handlungen, die füreinander erreichbar sind“ (Luhmann 1973, S. 83) verstanden. Offenbar ist dies nicht als wissenschaftlich begründbare These, sondern als unumstößliche Wahrheit zu verstehen: „Die Tatsache eines weltweiten Kommunikationssystems kann nicht bestritten werden.“ (Luhmann 1998, S. 373). Sie ist schlichtweg ein „Faktum“ (Stichweh 2000, S. 12). 
Die „Netzwerkgesellschaft“ (Castells 2001) beruht auf dem grundlegenden Gedanken einer weltweiten Konnektivität, die sich durch die neuen Kommunikations- und Informationstechnologien herstellen lässt. Die Bildung von Netzwerken resultiert aus den Verflechtungen und Verbindungen von bisher unverbundenen Elementen, durch die ehemals relevante Grenzen unterlaufen oder gar aufgehoben werden. Netze trennen nicht, Netze verbinden. Aufgrund dieser Qualität tragen sie zur „Intensivierung weltweiter sozialer Beziehungen“ (Giddens 1995, S. 85) bei und erfüllen damit das grundlegende Versprechen der Globalisierung und der Weltgesellschaft, „weltweite Interaktion“ (Luhmann 1975, S. 53) und „weltweite Verflechtungen“ (ebd., S. 54) über alle Grenzen hinweg zu ermöglichen - jenseits nationalstaatlicher Vorgaben und Trennlinien. Manuel Castells geht in seiner Konzeption einer Netzwerkgesellschaft von der „Auflösung der Orte im Raum der Ströme“ aus (Castells 2001, S. 475): „Ströme von Kapital, Ströme von Informationen, Ströme von Technologie, Ströme von organisatorischer Interaktion, Ströme von Bildern, Tönen und Symbolen“ (Castells 2003, S. 402) überwinden mühelos die Ebene des Lokalen und bilden eine neue globale Gesellschaftsstruktur.

Die raum- und grenzüberwindenden Konzeptionen der Welt- und Netzwerkgesellschaft finden in Zygmunt Baumans Diagnose der "flüchtigen Moderne“ (Bauman 2003), die an die Stelle der „schweren Moderne“ (ebd., S. 136) tritt, noch eine Steigerung. Bauman zufolge ist das gegenwärtige Zeitalter von einer umfassenden Verflüssigung des Sozialen gekennzeichnet: Liquid Modernity (Bauman 2000), Liquid Life (Bauman 2005), Liquid Fear (Bauman 2006), Liquid Times (Bauman 2007) lauten die Titel einiger seiner in den ersten Jahren des 21. Jahrhunderts veröffentlichten Bücher. In all diesen Publikationen wird im Grunde die immer gleiche Botschaft verbreitet: Das Feste weicht dem Flüssigen! „Raum, Grund und Boden waren zentrale Obsessionen der Moderne, das Streben nach ihrem Erwerb geradezu zwanghaft und die Bewachung der Grenzen entwickelte sich zur allgegenwärtigen, sich immer weiter verbreitenden Sucht.“ (Bauman 2003, S. 136) Damit ist es nun vorbei. Die „flüchtige Moderne“ hat zur „Irrelevanz des Raums“ (ebd., S. 140) und zu seiner „Entwertung“ (ebd., S. 141) geführt. In ihr herrscht das Fluide, Flüssige und Flüchtige, das sich durch keinerlei Grenze mehr aufhalten lässt. Was heute zählt, ist die Geschwindigkeit, die Beweglichkeit und die Flexibilität. Die Bewohner der flüssigen Moderne sind keine „Landtreter“ mehr (Schmitt 1981, S. 7; vgl. Schroer 2001b), sondern nomadische Subjekte, Weltenbummler, immer unterwegs, heute hier, morgen dort, sich geschmeidig an immer neue Bedingungen anpassend und dabei auf Grenzen jeglicher Art keine Rücksicht mehr nehmen müssend. So schwer die Moderne also auch gewesen sein mag: Eine regelrechte „Verflüssigungswut“ (Bauman 2003, S. 12) hat deren starre Strukturen und stabil geglaubte Institutionen einfach hinweggespült!

\section{Grenzen der Entgrenzung}

Die Konzepte der Globalisierung, der Weltgesellschaft, der Netzwerkgesellschaft und der flüssigen Moderne sind überaus wirkmächtige Beschreibungen der Gegenwartsgesellschaften, die viele Jahre den soziologischen Diskurs über die gesellschaftliche Wirklichkeit bestimmt haben. Zu keinem Zeitpunkt jedoch sind sie unwidersprochen geblieben. Die Kritik an diesen Konzepten bezieht sich dabei vor allem auch auf ihre These von der wachsenden Indifferenz der neuen Gesellschaftsformen gegenüber physischen Räumen und territorialen Grenzen.

Das Konzept der Weltgesellschaft ist als „soziologische Fiktion“ (Wagner 1997), als „Unbegriff und Phantom“ (Tudyka 1989) bezeichnet und kritisiert worden. Dabei wird argumentiert, 
dass von einer bereits erfolgten Realisierung eines welteinheitlichen Gesellschaftssystems gar keine Rede sein könne, da nicht einmal die immer wieder als Paradebeispiel angeführte globale Weltwirtschaft tatsächlich weltweit agiere (vgl. Wagner 1999, S. 33). Bemerkenswert ist, dass Zweifel an der raumabstinenten und grenzneutralen Ausrichtung des Konzepts der Weltgesellschaft auch von ihren Verfechtern selbst geäußert werden. Entgegen des dezidiert nichträumlich angesetzten Gesellschaftsbegriffs der Luhmann'schen Systemtheorie werden insbesondere in seiner Thematisierung von Exklusionsphänomenen explizit räumliche Kategorien bemüht (vgl. Schroer 2006, S. 149ff.; 2001c). Statt auf eine stetig steigende Homogenisierung der Lebensbedingungen und Verhaltensstandards stößt er auf Favelas, Slums und andere vernachlässigte, von der Globalisierungsdynamik übergangene oder von ihr abgehängte Gebiete, die keine Anbindung an die weltumfassenden Kommunikationsströme erlangen und in „schwarzen Löchern“ (Stichweh 1997, S. 132) zu verschwinden drohen. Rudolf Stichweh konzediert, dass die räumliche Ausgrenzung bestimmter Bevölkerungsteile von der übrigen Bevölkerung „exterritoriale, für Fremde unbetretbare Räume“ schafft, „die mit der These der Weltgesellschaft nicht ohne weiteres harmonisierbar sind“ (ebd., S. 127). Entgegen der apodiktischen Behauptung einer unabhängig von Grenzen und Räumen funktionierenden Weltgesellschaft braucht es offenbar doch „räumliche Grenzen, an denen man die Bewegung von Körpern kontrollieren kann“ (Luhmann 1995, S. 260).

Auch die Netzwerkgesellschaft zieht hinsichtlich ihres Umgangs mit dem Grenzbegriff Kritik auf sich: „Wenn es auch treffend sein mag, die Konstitution gegenwärtiger Gesellschaft mit der Netzwerkmetapher zu beschreiben: Grenzen werden nicht obsolet, das territoriale Moment verschwindet nicht.“ (Kaufmann 2006, S. 36) Statt von einem bloßen Wegfall, ist vielmehr von einer Transformation der Grenzen auszugehen: „Lineare Grenzziehungen werden netzwerkartig umgebildet.“ (ebd., S. 37) Und diese Umbildung hat gravierende Auswirkungen, die sich mit Brian Massumi als umfassende Ausweitung von Kontrollpunkten beschreiben lassen: „Man kann sich frei bewegen. Doch nach ein paar Schritten lauert immer ein Kontrollpunkt. Sie sind überall. Sie sind fest in die soziale Landschaft integriert.“ (Massumi 2010, S. 49) Grenzkontrollen erfolgen demnach nicht mehr länger nur an den Rändern staatlicher Hoheitsgebiete, sondern können jederzeit an jedem Ort vorgenommen werden, erfolgen flächendeckend. Statt einer Abnahme ist damit eher eine Zunahme von Grenzkontrollen zu konstatieren, da sie flexibel gehandhabt und überraschend ausgeübt werden können. Für die Netzwerkforschung ist jedoch bezeichnend, dass sie die Frage der Grenzen erst nachträglich aufgeworfen hat (vgl. Häußling 2009). Der Aufbau von Netzen geriet zunächst allein unter dem Gesichtspunkt in den Blick, dass damit bisher isoliert existierende Elemente miteinander verbunden werden können - unbekümmert um die Frage der unterschiedlich verteilten Möglichkeiten der Partizipation aufgrund fehlender Infrastruktur, auf die Bruno Latour hinweist:

„Das Telefon mag noch so universell verbreitet sein, wir wissen dennoch sehr genau, daß wir neben einer Telefonleitung verschmachten können, wenn wir mit ihr nicht durch einen Anschluß und einen Telefonapparat verbunden sind. [...] Die elektromagnetischen Wellen verbreiten sich vielleicht überallhin, aber eine Antenne, ein Abonnement und ein Decoder sind trotzdem notwendig, um Canal Plus zu empfangen." (Latour 1998, S. 157, Herv. i. O.)

Obwohl sich daran bis heute nichts geändert hat, steckt die systematische Berücksichtigung der Infrastruktur als unverzichtbare Basis aller Kommunikationsnetzwerke noch in den Anfängen. 
George Ritzer hält dem Bauman'schen Konzept der flüchtigen Moderne und der behaupteten Ablösung des Festen durch das Flüchtige bzw. Flüssige entgegen, dass beide Aggregatzustände des Sozialen nicht einander ablösen, sondern in vielfältigen Kombinationen miteinander verbunden sind. Ihm geht es darum zu zeigen, „dass Flüssiges nie ohne feste Strukturen fließt“ (Ritzer 2002, S. 53). In Containern, Kanälen, Staudämmen, Blockaden, Sperren, Hürden und Sieben sieht er wirkungsvolle Begrenzungstechnologien am Werk, die das in Fluss geratene „umgeben, kanalisieren, eindämmen oder gar zu blockieren suchen“ (ebd., S. 73). Sie sorgen dafür, dass es zu keinem gänzlich ungehinderten und konturlosen Fließen kommt. Die „Festigkeit der Moderne“ (ebd.) erweist sich nach dieser Lesart als weitaus zählebiger als die Propheten eines neuen globalen Zeitalters suggerieren.

Das damit offenkundig nicht erst heute erkennbare Defizit vieler Theorien der Globalisierung, der Welt- und Netzwerkgesellschaft sowie der flüchtigen Moderne besteht grundsätzlich darin, Grenzen und Räume als Kategorien der Vergangenheit auszuweisen und zu verabschieden, sich aber kaum für die sich neu herausbildenden räumlichen Konfigurationen und neuen Grenzbildungsprozesse zu interessieren, die ihre Vorläufer zumeist keineswegs so restlos ablösen wie oftmals suggeriert wird. Entgegen der vorschnellen Verabschiedungsrhetorik in solchen und anderen soziologischen Zeitdiagnosen, drängt sich zudem die Persistenz bestimmter Raumund Grenzkonstellationen auf, die es stärker zu berücksichtigen gilt, als dies gemeinhin geschieht.

\section{Persistenz, Metamorphose und Ausweitung von Grenzen}

Angesichts der jüngsten weltgeschichtlichen Entwicklungen werden die schon zur Hochzeit der Globalisierung artikulierten Zweifel an den weitgehend raum- und grenzenlos gedachten Gesellschaftskonzeptionen nicht eben geringer. Ein aktueller Blick auf die Weltkarte zeigt, dass von einer grenzenlosen Welt gar keine Rede sein kann. Ganz im Gegenteil, wir sehen uns einer „very bordered world“ (Diener/Hagen 2012, S. 1) gegenüber. Der Traum von einer sich zunehmend verflechtenden und zusammenwachsenden Welt scheint - zumindest vorerst ausgeträumt. Wer wollte der vor kaum mehr als zehn Jahren publizierten Diagnose Baumans heute noch zustimmen:

„Der Druck auf die Grenzen, der darauf abzielt, sie zu durchlöchern und zu demontieren, und den man gemeinhin ,Globalisierung' nennt, hat Wirkung gezeigt: Mit wenigen Ausnahmen, deren Anzahl rapide sinkt, stehen heute die Tore aller Gesellschaften weit offen, materiell wie intellektuell.“ (Bauman 2008, S. 14)

Entgegen dieser bemerkenswert optimistischen Einschätzung muss heute eine wahre Renaissance der Grenzen und Wiederaufrüstung von Grenzanlagen konstatiert werden (vgl. Leuenberger in diesem Band). Die Liste der derzeit wieder aufgerüsteten, neu gebauten und in Planung befindlichen Grenzbefestigungen ist lang (vgl. Thränhardt 2012; Debray 2016). Sie reicht von der geplanten Mauer zwischen den Vereinigten Staaten und Mexiko über die Sperranlage zwischen Israel und Palästina bis hin zu den innerhalb Europas eilig errichteten Grenzzäunen als politische Antwort auf die so genannte „Flüchtlingskrise“. Das „gegenwärtige nationalstaatliche Mauerbaufieber" (Brown 2018, S. 46) greift offenbar um sich und droht alle Länder auf dem Planeten Erde in streng gegeneinander abgeschirmte Festigungsanlagen zu verwandeln. 
Träfe diese Beschreibung vollends zu, hätten wir es nach einer Zeit der vor allem in den 1990er Jahren verbreiteten Diagnosen der Öffnung mit einer radikalen Umkehrbewegung zu tun, die uns eine Rückkehr ins Geschlossene beschert. Zweifellos lassen sich für die These einer neuen „Abschottung“ (Brown 2018; Marshall 2018) eine stattliche Anzahl von Indizien anführen, die kaum von der Hand zu weisen sind: Das Wiedererstarken des Nationalismus, die Erfolge des Rechtspopulismus, die restriktive Migrationspolitik, der neue Protektionismus und die überall aus dem Boden sprießenden Zäune und Mauern sprechen eindeutig dafür. Am Ende aber könnte sich eine solche Diagnose als ebenso eindimensional erweisen wie die zahlreichen Prognosen, die uns noch vor gar nicht langer Zeit einen „Umzug ins Offene“ (Fecht/Kamper 2000) versprochen haben. Doch so wenig der Nationalstaat durch die Globalisierung tatsächlich abgelöst wurde, so wenig kehrt er nun in alter Pracht zurück, so als seien die Entwicklungen spurlos an ihm vorübergegangen. Um einer solch auf den ersten Blick naheliegenden, letztlich aber zu kurz greifenden Rollback-These zu entkommen, erweist sich gerade der nüchtern analysierende Blick auf Grenzen als hilfreiches Antidot gegen verabsolutierende Trendaussagen, deren Halbwertszeit ohnehin immer kürzer zu werden scheinen. Denn nur dem - medial massiv unterstützten - Anschein nach, haben wir es mit einer bloßen Wiederkehr der aus Mauern und Stacheldraht errichteten Befestigungsanlagen im klassischen Gewand zu tun, die das Bild einer Rückkehr in durch streng bewachte Grenzen strikt voneinander geschiedenen Nationalstaaten heraufbeschwören. Was dieses Bild letztlich erzeugt, ist der einseitige Blick auf die nationalstaatliche Grenze als Grenzlinie, der insofern gleich mehrfach trügt, weil bei der Erschließung der vormodernen Grenzverhältnisse, beim Blick auf die netzwerkartigen globalen Strukturen und bei der heutigen Wiederkehr der Grenzbefestigungsanlagen durchgehend diese spezielle Form der Grenze als Maßstab genommen wird, die wir von der Trennung zweier Nationalstaaten in der Fläche her kennen. Nur wenn diese zum Idealbild einer Grenze erhoben wird, gab es territoriale Grenzen - entgegen unserer eigenen Rekonstruktion - in vormodernen Gesellschaften noch kaum (vgl. Parsons 1975, S. 65), während der großen Zeit der Nationalstaaten nahezu durchgängig, in der Ära der Globalisierung nicht mehr oder kaum noch und aktuell wieder in großem Ausmaß. Statt ein solches Auf- und Abtreten des immer gleichen Grenztyps in immer neuen gesellschaftlichen Formationen zu rekonstruieren, wäre es im Sinne einer elaborierten Grenzforschung weitaus gewinnbringender, den Wandel der Grenzen und der Grenzauffassungen selbst stärker ins Auge zu fassen, als dies zumeist der Fall ist. Die Phänomenologie von Grenzen erweist sich dabei als überaus reichhaltig. Wer dagegen ihr Verschwinden prognostiziert oder ihre Wiederkehr verkündet, dem entgeht das Entscheidende: die Metamorphosen und der Formwandel von Grenzen. Nimmt man diese in den Blick, erweisen sich die Anzeichen einer Wiederkehr der nationalstaatlichen Grenzarchitektur als zumindest trügerisch, denn die aktuellen Grenzanlagen bestehen aus der Akkumulation von sowohl alten wie neuen, harten wie weichen Kontroll- und Überwachungstechnologien, also aus Mauern, Zäunen, Stacheldrahtrollen und Wachtürmen ebenso wie aus Drohnen, Hubschraubern, Wärmebildkameras und Nachtsichtgeräten (vgl. auch Nail in diesem Band). Hinzu kommen Pässe, Visa, Gesundheitszertifikate, Zoll- und Finanzbeamte, Gesundheits- und Einwanderungsbehörden. Kurz: Es kommt alles zum Einsatz, was die Menschen im Laufe des gesellschaftlichen Werdens an Begrenzungstechnologien erfunden haben - mixed borders with mixed materials gewissermaßen. Durch die Mischung aus sichtbaren und unsichtbaren, diskursiven und nichtdiskursiven Elementen lässt sich die Grenze in ihrer aktuellen Form treffend als „Raumdispositiv“ (Walters 2011, S. 322) oder „Grenzregime“ (Opitz 2011, S. 259) beschreiben. Im Gegensatz zum naheliegenden Befund eines martialischen Aufbaus von Grenzbefesti- 
gungsanlagen zur Verwirklichung einer totalen Abschottung ist vielmehr die Perfektionierung der Grenze als Selektionsinstrument das Ziel der massiven Aufrüstung ausgefeilter Grenzapparaturen (vgl. Schroer 2019b). Entgegen des äußeren Anscheins sollen die hoch technologisierten Grenzarchitekturen nicht für die lückenlose Abdichtung der Grenzen, sondern für einen geregelten Grenzverkehr sorgen und damit die genuine Funktion der Grenze als Instrument der Selektion erfüllen, denn die Bestrebungen gehen offenbar grundsätzlich dahin, allen unerwünschten Migrationen mit Schließung und allen erwünschten mit Öffnung der Grenzen zu begegnen, den Zustrom von Waren und Personen also nicht grundsätzlich zu unterbinden, sondern zu kanalisieren und zu filtern (vgl. Hess/Schmidt-Sembdner in diesem Band). Die Souveränität eines Staates wird in Zukunft wohl mehr denn je daran festgemacht werden, ob er zu einem solchen Sortierungsvorgang auf der Höhe der technologischen Möglichkeiten in der Lage ist oder nicht (vgl. dazu Pötzsch in diesem Band). Allerdings geht es nicht nur um die vom Staat gezogenen territorialen Grenzen, sondern auch um die von anderen sozialen Einheiten gezogenen. Nicht nur jeder Staat, auch jede Stadt, jede Organisation, jede soziale Gruppe und jedes Individuum zieht Grenzen und steckt damit Räume ab, die von den einen betreten werden dürfen und von den anderen nicht. Dabei geht es um die Aneignung und Besetzung von Territorien - von den „Territorien des Selbst“ (Goffman 1974) über städtische Territorien bis zu staatlichen Territorien. Wer Kämpfe um Territorien für ein vormodernes oder gar nur der Tierwelt vorbehaltenes, zumindest aber für ein die freischwebenden Netze der Weltgesellschaft in ihrer Selbstgenügsamkeit nicht tangierendes Phänomen gehalten hat, wird zur Kenntnis nehmen müssen, dass wir es auch weiterhin und offenbar mit zunehmender Intensität mit der Besetzung und Verteidigung von Territorien zu tun haben werden, bei dem es nicht um das einmalige Ziehen und Festlegen von Grenzen geht, sondern um sich permanent vollziehende Grenzpraktiken und den immer neuen Kampf um den Verlauf von Grenzen.

\section{Fazit}

Unsere Überlegungen über das Verhältnis zwischen Gesellschaft und Grenze haben gezeigt, dass die Entwicklung von Gesellschaften ohne die Berücksichtigung der Grenzen, die sie für sich bestimmt, kaum umfassend darstellbar ist, denn gänzlich ohne Grenzen kommt keine Gesellschaft aus. Aber statt von der sukzessiven Etablierung oder dem stetigen Abbau von Grenzen zu erzählen, ist der Formwandel der Grenzen stärker als bisher in den Blick zu nehmen. Von der Ackerfurche über den Zaun und die Mauer bis zur Firewall durchziehen Grenzkonstruktionen die Geschichte der Menschheit. Über die Art und Weise ihrer Grenzziehungen konstituieren sich Gesellschaften zu einem je spezifischen Gebilde. Eine Jäger- und Sammlergesellschaft ist durch andere Grenzen charakterisiert als eine Netzwerkgesellschaft. Den jeweiligen „Gesellschaftstypen“ (Tenbruck 1989a, S. 59) können deshalb entsprechende Grenztypen hinzugefügt werden. Welche Grenzziehungen jeweils vorgenommen werden, sagt viel über die jeweilige Gesellschaftsform aus, mit der wir es zu tun haben. Auf diese Weise ließe sich auch der von Tenbruck vorgeschlagene Gesellschaftsbegriff durchaus auch weiterhin fruchtbar als Grundlage soziologischen Denkens einsetzen:

„Anstatt die Existenz der ,Gesellschaft‘ vorauszusetzen, ist es die Aufgabe der Soziologie zu prüfen, ob, wann, wie und warum aus vielen Vergesellschaftungen eine Gesellschaft entsteht. Insofern ist die Gesellschaft nicht der gegebene Ausgangspunkt, sondern das aufgegebene Rätsel der Soziologie.“ (Tenbruck 1989b, S. 429) 
Ein fruchtbarer methodischer Weg, dieser von Latour (2007) verblüffend ähnlich formulierten Aufgabe nachzukommen, bestünde darin, die jeweils vorgenommenen Grenzziehungen systematisch nachzuverfolgen, die jede Vergesellschaftung begleiten.

\section{Weiterführende Literatur}

Agier, Michel (2016): Borderlands. Towards an Anthropology of the Cosmopolitan Condition. New York: John Wiley and Sons.

Bredow, Wilfried von (2014): Grenzen. Eine Geschichte des Zusammenlebens vom Limes bis Schengen. Darmstadt: Wissenschaftliche Buchgesellschaft.

Diener, Alexander C./Hagen, Joshua (2012): Borders. A Very Short Introduction. Oxford: University Press. Eigmüller, Monika/Vorbuba, Georg (Hrsg.) 2016: Grenzsoziologie. Die politische Strukturierung des Raumes. 2. Aufl. Wiesbaden: VS.

Foucher, Michel (2007): L' Obsession des frontiers. Paris: Librairie Académique Perrin.

\section{Literaturverzeichnis}

Albrow, Martin (1998): Abschied vom Nationalstaat. Frankfurt/M.: Suhrkamp.

Bauman, Zygmunt (2000): Liquid Modernity. Cambridge: Polity Press.

Bauman, Zygmunt (2003): Flüchtige Moderne. Frankfurt/M.: Suhrkamp.

Bauman, Zygmunt (2005): Liquid Life. Cambridge: Polity Press.

Bauman, Zygmunt (2006): Liquid Fear. Cambridge: Polity Press.

Bauman, Zygmunt (2007): Liquid Times. Living in an Age of Uncertainty. Cambridge: Polity Press.

Beck, Ulrich (1997): Was ist Globalisierung? Frankfurt/M.: Suhrkamp.

Bös, Mathias (2000): Zur Kongruenz sozialer Grenzen. Das Spannungsfeld von Territorien, Bevölkerungen und Kulturen in Europa. In: Bach, Maurizio (Hrsg.): Die Europäisierung nationaler Gesellschaften. Wiesbaden: VS 2000, S. 429-455.

Bös, Mathias/Zimmer, Kerstin (2006): Wenn Grenzen wandern. Zur Dynamik von Grenzverschiebungen im Osten Europas. In: Eigmüller, Monika/Vorbuba, Georg (Hrsg.): Grenzsoziologie. Die politische Strukturierung des Raumes. Wiesbaden: VS, S. 157-184.

Brown, Wendy (2018): Mauern. Die neue Abschottung und der Niedergang der Souveränität. Berlin: Suhrkamp.

Bühler, Benjamin (2014): Grenzstein. In: Ders./Rieger, Stefan (Hrsg.): Bunte Steine. Ein Lapidarium des Wissens. Berlin: Suhrkamp 2014, S. 69-80.

Castells, Manuel (2001): Das Informationszeitalter, Bd. 1: Der Aufstieg der Netzwerkgesellschaft. Opladen: Leske + Budrich.

Castells, Manuel (2003): Das Informationszeitalter, Bd. 3: Jahrtausendwende. Opladen: Leske + Budrich.

Castoriadis, Cornelius (1984): Gesellschaft als imaginäre Institution. Entwurf einer politischen Philosophie. Frankfurt/M.: Suhrkamp.

Castoriadis, Cornelius (2010): Das Imaginäre: die Schöpfung im gesellschaftlich-geschichtlichen Bereich. In: Ders.: Das imaginäre Element und die menschliche Schöpfung. Lich: Edition AV, S. 25-45.

Claessens, Dieter (1980): Das Konkrete und das Abstrakte. Soziologische Skizzen zur Anthropologie. Frankfurt/M.: Suhrkamp.

Coulanges, Fustel de (1996): Der antike Staat. Studie über Kultus, Recht und Einrichtungen Griechenlands und Roms. Essen: Athenaion.

Debray, Regis (2016): Lob der Grenzen. Hamburg: Laika.

Delitz, Heike (2010): Die gebaute Gesellschaft. Architektur als Medium des Sozialen. Frankfurt/M./New York: Campus.

Diener, Alexander C./Hagen, Joshua (2012): Borders. A Very Short Introduction. Oxford: Oxford University Press.

Durkheim, Émile (1981): Einführung in die Sozialwissenschaft. Eröffnungsvorlesung von 1887-1888. In: Ders.: Frühe Schriften zur Begründung der Sozialwissenschaft. Herausgegeben, eingeleitet und übersetzt von Lore Heisterberg. Darmstadt und Neuwied, S. 25-52.

Durkheim, Émile (1991): Physik der Sitten und des Rechts. Vorlesungen zur Soziologie der Moral. Frankfurt/M.: Suhrkamp.

Durkheim, Émile (2010): Notiz über Soziale Morphologie (Auszug). In: Marcel Mauss: Soziologie und Anthropologie, Bd. 1: Theorie der Magie/Soziale Morphologie. Wiesbaden: VS, S. 182.

Eßbach, Wolfgang (1999): Anthropologische Überlegungen zum Begriff der Grenze in der Soziologie. In: Fludernik, Monika/Gehrke, Hans-J. (Hrsg.): Grenzgänger zwischen Kulturen. Würzburg, S. 85-98. 
Fecht, Tom/Kamper, Dietmar (2000): Umzug ins Offene. Vier Versuche über den Raum. Wien/New York: Springer Verlag.

Flusser, Vilém (2006): Räume. In: Dünne, Jörg/Günzel, Stephan (Hrsg.): Raumtheorie. Grundlagentexte aus Philosophie und Kulturwissenschaften. Frankfurt/M.: Suhrkamp, S. 274-285.

Freyer, Hans (1965): Schwelle der Zeiten. Beiträge zur Soziologie der Kultur. Stuttgart: DVA.

Gehlen, Arnold (1964): Die Sozialstrukturen primitiver Gesellschaften. In: Ders./Schelsky, Helmut (Hrsg.): Soziologie. Lehr- und Handbuch zur modernen Gesellschaftskunde. 5.Aufl., Düsseldorf: Dieterichs.

Giddens, Anthony (1995): Konsequenzen der Moderne. Frankfurt/M.: Suhrkamp.

Goffman, Erving (1974): Territorien des Selbst. In: Ders.: Das Individuum im öffentlichen Austausch. Frankfurt/M.: Suhrkamp, S. 54-96.

Habermas, Jürgen (1998): Die postnationale Konstellation. Politische Essays. Frankfurt/M.: Suhrkamp.

Halbwachs, Maurice (2009): Soziale Morphologie. Konstanz: UVK.

Häußling, Roger (Hrsg.) 2009: Grenzen von Netzwerken. Wiesbaden: VS.

Immerfall, Stefan (1995): Einführung in den europäischen Gesellschaftsvergleich. Ansätze - Problemstellungen - Befunde. 2. erg. und überarb. Aufl., Passau: Wissenschaftsverlag Rothe.

Kaufmann, Stefan (2006): Grenzregimes im Zeitalter sozialer Netzwerke. In: Berking, Helmuth (Hrsg.): Die Macht des Lokalen in einer Welt ohne Grenzen. Frankfurt/M./New York: Campus, S. 32-65.

Klotz, Heinrich (1991): Von der Urhütte bis zum Wolkenkratzer. Geschichte der gebauten Umwelt. München: Prestel.

Latour, Bruno (1998): Wir sind die modern gewesen. Frankfurt/M.: Fischer.

Latour, Bruno (2007): Eine neue Soziologie für eine neue Gesellschaft. Frankfurt/M.: Suhrkamp.

Leroi-Gourhan, André (1980): Hand und Wort. Die Evolution von Technik, Sprache und Kunst. Frankfurt/M.: Suhrkamp.

Lévi-Strauss, Claude (1967): Strukturale Anthropologie I. Frankfurt/M.: Suhrkamp.

Lévi-Strauss, Claude (1975): Strukturale Anthropologie II. Frankfurt/M.: Suhrkamp.

Lindemann, Gesa (2004): Reflexive Anthropologie und die Analyse des Grenzregimes. Zur Aktualität Helmuth Plessners. In: Bröckling, Ulrich; Bühler, Benjamin; Hahn, Marcus; Schöning, Matthias; Weinberg, Manfred (Hrsg.): Disziplinen des Lebens. Zwischen Anthropologie, Literatur und Politik. Tübingen: Narr, S. 23-34.

Lotman, Jurij (2010): Der Begriff der Grenze. In: Ders.: Die Innenwelt des Denkens. Eine semiotische Theorie der Kultur. Frankfurt/M.: Suhrkamp, S. 174-189.

Løvschal, Mette (2014): Frühe Grenzziehungen. In: Aus Politik und Zeitgeschichte 4-5, S. 19-25.

Luhmann, Niklas (1971): Die Weltgesellschaft. In: Ders.: Soziologische Aufklärung, Bd. 1. Opladen: Westdeutscher Verlag 1975, S. 51-71.

Luhmann, Niklas (1973): Selbst-Thematisierung des Gesellschaftssystems. Über die Kategorie der Reflexion aus der Sicht der Systemtheorie. In: Ders.: Soziologische Aufklärung, Bd. 2. Opladen: Westdeutscher Verlag 1975, S. 72-102.

Luhmann, Niklas (1994): Die Unterscheidung von Staat und Gesellschaft. In: Ders.: Soziologische Aufklärung, Bd. 4. Opladen: Westdeutscher Verlag, S. 67-73.

Luhmann, Niklas (1997): Die Gesellschaft der Gesellschaft. Frankfurt/M.: Suhrkamp.

Luhmann, Niklas (1998): Der Staat des politischen Systems. In: Beck, Ulrich (Hrsg.): Perspektiven der Weltgesellschaft. Frankfurt/M.: Suhrkamp, S. 345-380.

Malinowski, Boris (2005): Eine wissenschaftliche Theorie der Kultur. Frankfurt/M.: Suhrkamp 1975.

Marshall, Tim (2018): Abschottung. Die neue Macht der Mauern. München: dtv.

Massumi, Brian (2010): Bewegungen navigieren. In: Ders: Ontomacht. Kunst, Affekt und das Ereignis des Politischen. Berlin: Merve, S. 25-67.

Mauss, Marcel (2010): Über den jahreszeitlichen Wandel der Eskimogesellschaften. Eine Studie zur Sozialen Morphologie. In: Ders: Soziologie und Anthropologie, Bd. 1: Theorie der Magie/Soziale Morphologie. Wiesbaden: VS, S. 183-278.

Mauss, Marcel (2017): Die Nation oder der Sinn für das Soziale. Frankfurt/M./New York: Campus.

Ohmae, Kenichi (1990): The Borderless World. Power and strategy in the interlinked economy, New York: Harper Business.

Opitz, Sven (2001): Stichwort „Grenzregime“. In: Fuchs-Heinritz, Werner/Klimke, Daniela; Lautmann, Rüdiger, Rammstedt, Otthein, Stäheli, Urs; Weischer, Christoph, Wienold, Hanns (Hrsg.): Lexikon zur Soziologie. Wiesbaden: VS.

Parsons, Talcott (1975): Gesellschaften. Evolutionäre und komparative Perspektiven. Frankfurt/M.: Suhrkamp.

Popitz, Heinrich (1995): Der Aufbruch zur Artifiziellen Gesellschaft. Zur Anthropologie der Technik. Tübingen: J. C. B. Mohr. 
Raffestin, Claude (2010): Elemente einer Theorie der Grenze. In: Ders.: Zu einer Geographie der Territorialität. Stuttgart: Franz Steiner, S. 57-72.

Rehberg, Karl-Siegbert (1985): Kultur versus Gesellschaft? Anmerkungen zu einer Streitfrage in der deutschen Soziologie. In: Moebius, Stephan/Albrecht, Clemens (Hrsg.): Kultursoziologie. Klassische Texte der neueren deutschen Kultursoziologie. Wiesbaden: Springer VS, 2014, S. 367-396.

Rehberg, Karl-Siegbert (2008): Die Natur der Gesellschaft. Verhandlungen des 33. Kongresses der Deutschen Gesellschaft für Soziologie in Kassel 2006. Frankfurt/M./New York: Campus.

Ritzer, George (2002): Festes in einer Welt des Flusses. Die Beständigkeit der Moderne in einer zunehmend postmodernen Welt. In: Junge, Matthias/Kron, Thomas (Hrsg.): Zygmunt Bauman. Soziologie zwischen Postmoderne und Ethik. Opladen: Leske + Budrich, S. 51-79.

Robertson, Ronald (1992): Globalization. Social Theory and Global Culture. London: Routledge.

Rokkan, Stein (2000): Staat, Nation und Demokratie. Frankfurt/M.: Suhrkamp.

Sassen, Saskia (2008): Das Paradox des Nationalen. Territorialität, Autorität und Rechte im globalen Zeitalter. Frankfurt/M.: Suhrkamp.

Scheuerbrandt, Jörg (2009): Der Limes. In: Linn, Astrid (Hrsg.): Mauern als Grenzen. Mainz am Rhein: Philipp von Zabern, S. 93-107.

Schmitt, Carl (1981): Land und Meer. Eine weltgeschichtliche Betrachtung. Köln-Lövenich: Klett-Cotta.

Schmitt, Carl (1997): Der Nomos der Erde im Völkerrecht des Jus Publicum Europaeum. Berlin: Duncker \& Humblot.

Schroer, Markus (2001a): Das Individuum der Gesellschaft. Synchrone und diachrone Theorieperspektiven. Frankfurt/M.: Suhrkamp.

Schroer, Markus (2001b): Land und (Daten)meer. Zur Raumaneignung im Internet. In: Telepolis. Magazin der Netzkultur. www.heise-news.de/tp/deutsch/inhalt/co/9345/1.html 28.02.2020.

Schroer, Markus (2001c): Die im Dunkeln sieht man doch? Inklusion/Exklusion und die Entdeckung der „Überflüssigen“. In: Mittelweg 36 10, H. 5, S. 33-46.

Schroer, Markus (2006): Räume, Orte, Grenzen. Auf dem Weg zu einer Soziologie des Raums. Frankfurt/M.: Suhrkamp.

Schroer, Markus (2014): Visual Culture and the Fight for Visibility. In: Journal for the Theory of Social Behaviour 44 (2), S. 206-228.

Schroer, Markus (2018a): Soziale Morphologie. In: Kopp, Johannes/Steinbach, Anja (Hrsg.): Grundbegriffe der Soziologie. Wiesbaden: VS, S. 331-336.

Schroer, Markus (2018b): Zwischen Öffnung und Abschottung: Grenzen in einer globalisierten Welt. In: Draiflessen Collection (Hrsg.): Grenzüberschreitend. Katalog zur Ausstellung. Mettingen: Draiflessen Collection, S. 75-82.

Schroer, Markus (2019a): Grenzen - ihre Bedeutung für Stadt und Architektur. In: Ders.: Räume der Gesellschaft. Soziologische Studien. Wiesbaden: Springer VS, S. 247-256.

Schroer, Markus (2019b): Grenzen - ihre Dissemination und Diversifizierung. In: Ders.: Räume der Gesellschaft. Soziologische Studien. Wiesbaden: Springer VS, S. 257-265.

Schroer, Markus (i. E.): The Sociality of Space. In: Joas, Hans/Pettenkofer, Andreas (Hrsg.): Oxford Handbook of Emile Durkheim. Oxford: University Press.

Service, Elman R. (1977): Ursprünge des Staates und der Zivilisation. Der Prozess der kulturellen Evolution. Frankfurt/M.: Suhrkamp.

Simmel, Georg (1992): Soziologie. Untersuchungen über die Formen der Vergesellschaftung. Gesamtausgabe Bd. 11, Hrsg. von Otthein Rammstedt. Frankfurt/M.: Suhrkamp.

Stichweh, Rudolf (1997): Inklusion/Exklusion, funktionale Differenzierung und die Theorie der Weltgesellschaft. In: Soziale Systeme 3, S. 123-136.

Stichweh, Rudolf (2000): Die Weltgesellschaft. Soziologische Studien. Frankfurt/M.: Suhrkamp.

Tenbruck, Friedrich (1989a): Gesellschaft und Gesellschaften: Gesellschaftstypen. In: Ders.: Die kulturellen Grundlagen der Gesellschaft. Opladen: Westdeutscher Verlag, S. 59-79.

Tenbruck, Friedrich (1989b): Gesellschaftsgeschichte oder Weltgeschichte? In: Kölner Zeitschrift für Soziologie und Sozialpsychologie 41, S. 417-439.

Thränhardt, Dietrich (2012): Neue Grenzen in der Globalisierung. Warum Staaten wieder Mauern bauen. In: INDES. Zeitschrift für Politik und Gesellschaft, H. 4/2012, S. 14-22.

Tönnies, Ferdinand (1979): Gemeinschaft und Gesellschaft. Darmstadt: Wissenschaftliche Buchgesellschaft.

Trebsche, Peter/Müller-Scheeßel, Nils/Reinhold, Sabine (2010): Einleitung. In: Dies. (Hrsg.): Der gebaute Raum. Bausteine einer Architektursoziologie vormoderner Gesellschaften. Münster: Waxmann, S. 9-28.

Trier, Jost (1943): Zaun und Mannring. In: Beiträge zur Geschichte der deutschen Sprache und Literatur 66 , S. 232-264. 
Tudyka, Kurt (1989): Weltgesellschaft - Unbegriff und Phantom. In: Politische Vierteljahresschrift 30, S. 503-508.

Tyrell, Hartmann (2005): Singular oder Plural - Einleitende Bemerkungen zu Globalisierung und Weltgesellschaft. In: Heintz, Bettina/Münch, Richard/Ders. (Hrsg.): Weltgesellschaft. Theoretische Zugänge und empirische Problemlagen. Stuttgart: Lucius \& Lucius, S. 1-50.

Uzarewicz, Michael (2016): Der Leib und die Grenzen der Gesellschaft: Eine neophänomenologische Soziologie des Transhumanen. Stuttgart: Lucius \& Lucius.

Wagner, Gerhard (1999): Herausforderung Vielfalt. Plädoyer für eine kosmopolitische Soziologie. Konstanz: UVK.

Walters, William (2011); Mapping Schengenland. In: Piper, Marianne (Hrsg.): Biopolitik - in der Debatte. Wiesbaden: VS, S. 305-337. 